Bull. Korean Math. Soc. 50 (2013), No. 1, pp. 263-273

http://dx.doi.org/10.4134/BKMS.2013.50.1.263

\title{
MEAN-VALUE PROPERTY AND CHARACTERIZATIONS OF SOME ELEMENTARY FUNCTIONS
}

\author{
JANUSZ MATKOWSKI
}

ABSTRACT. A mean-value result, saying that the difference quotient of a differentiable function in a real interval is a mean value of its derivatives at the endpoints of the interval, leads to the functional equation

$$
\frac{f(x)-F(y)}{x-y}=M(g(x), G(y)), \quad x \neq y,
$$

where $M$ is a given mean and $f, F, g, G$ are the unknown functions. Solving this equation for the arithmetic, geometric and harmonic means, we obtain, respectively, characterizations of square polynomials, homographic and square-root functions. A new criterion of the monotonicity of a real function is presented.

\section{Introduction}

In a recent paper [4] the following counterpart of the Lagrange mean-value theorem has been proved. If a real function $f$ defined on an interval $I \subset \mathbb{R}$ is differentiable, and $f^{\prime}$ is one-to-one, then there exists a unique mean function $M: f^{\prime}(I) \times f^{\prime}(I) \rightarrow f^{\prime}(I)$ such that

$$
\frac{f(x)-f(y)}{x-y}=M\left(f^{\prime}(x), f^{\prime}(y)\right), \quad x, y \in I, x \neq y .
$$

One can show [5] that, in this equality, $M$ is a power mean if and only if one of the following cases occurs:

$M$ is the arithmetic mean, that is $M=\mathcal{A}$ where

$$
\mathcal{A}(u, v)=\frac{u+v}{2}, \quad u, v \in \mathbb{R},
$$

and the function $f$ is a quadratic polynomial;

$M$ is the geometric, that is $M=\mathcal{G}$ where

and $f$ is a homographic function;

$$
\mathcal{G}(u, v)=\sqrt{u v}, \quad u, v>0,
$$

Received July 28, 2011

2010 Mathematics Subject Classification. Primary 39B22, 26A24, 26A48.

Key words and phrases. mean-value theorem, classical means, monotonic functions, quadratic function, homographic function, square root function, functional equation. 
$M$ is the harmonic, that is $M=\mathcal{H}$ where

$$
\mathcal{H}(u, v)=\frac{2 u v}{u+v}, \quad u, v>0,
$$

and $f$ is the square root of an affine function.

In the present paper, assuming that $M \in\{\mathcal{A}, \mathcal{G}, \mathcal{H}\}$, we consider the functional equation

$$
\frac{f(x)-F(y)}{x-y}=M(g(x), G(y)), \quad x, y \in I, x \neq y,
$$

where all functions $f, F, g, G: I \rightarrow \mathbb{R}$ are unknown.

In the auxiliary Section 1 we first observe if the real functions $f, F$ defined on a set $I \subset R$ of the cardinality grater than 2 satisfy the inequality

$$
\frac{f(x)-F(y)}{x-y} \geq 0, \quad x, y \in I, x \neq y,
$$

then both functions are nondecreasing. Moreover, if $I$ is an interval, the function $f$ and $F$ coincide at the continuity points of these functions (Theorem 1). This new criterion of monotonicity appears to be very helpful in proving of the main results.

In Section 2, assuming that $M=\mathcal{A}$, we show that (without any regularity assumptions) the functions $f, F, g, G$ satisfy the equation (*) if and only if the functions $f$ and $F$ are equal to a quadratic polynomials and the functions $g=G$ is its derivative (Theorem 2).

In Section 3 we consider the case $M=\mathcal{G}$. We show that $f, F, g, G$ satisfy the equation $(*)$ if and only if $f=F$ is either affine or homographic, and the functions $g$ and $G$, up to a multiplicative constant, are equal to the derivative of $f$ (Theorem 3 ).

In Section 4, assuming that $M=\mathcal{H}$, we prove that $f, F, g, G$ satisfy the equation $(*)$ if and only if $f=F$ is either affine or square root of an affine function, and $g=G$ is the derivative of $f$ (Theorem 4).

The idea of this paper is due to J. Aczél [1], who characterized the quadratic polynomials using only the mean value property of their derivatives (cf. also M. Kuczma [3] and J. Aczél and M. Kuczma [2]).

\section{Difference quotient and a criterion of monotonicity}

Lemma 1. Let $I \subset \mathbb{R}$ be an arbitrary set such that card $I \geq 3$. If the functions $f, F: I \rightarrow \mathbb{R}$ satisfy the inequality

$$
\frac{f(x)-F(y)}{x-y} \geq 0, \quad x, y \in I, x \neq y,
$$

then, for all $x_{1}, x_{2}, x_{3} \in I$ such that $x_{1}<x_{2}<x_{3}$,

$$
f\left(x_{1}\right) \leq F\left(x_{2}\right) \leq f\left(x_{3}\right) \quad \text { and } \quad F\left(x_{1}\right) \leq f\left(x_{2}\right) \leq F\left(x_{3}\right) ;
$$

in particular, the functions $f$ and $F$ are nondecreasing. 
If inequality (1.1) is sharp, then inequalities (1.2) are sharp and $f, F$ are strictly increasing.

Proof. Let $x_{1}, x_{2}, x_{3} \in I$ be such that $x_{1}<x_{2}<x_{3}$. Taking $x:=x_{1}$ and $y:=x_{2}$ in (1.1) we get $f\left(x_{1}\right) \leq F\left(x_{2}\right)$ and taking $x:=x_{3}$ and $y:=x_{2}$ in (1.1) we get $F\left(x_{2}\right) \leq f\left(x_{3}\right)$ whence $f\left(x_{1}\right) \leq F\left(x_{2}\right) \leq f\left(x_{3}\right)$. Similarly, taking first $x:=x_{2}$ and $y:=x_{1}$; then $x:=x_{3}$ and $y:=x_{2}$ we get the remaining inequalities in $(1.2)$.

To show that the assumption card $I \geq 3$ is indispensable, consider the following:

Example 1. Take $I:=\{1,2\}$ and $f, F: I \rightarrow \mathbb{R}$ defined by $f(1)=3, f(2)=2$ and $F(1)=1, F(2)=4$. Then

$$
\frac{f(1)-F(2)}{1-2}=\frac{f(2)-F(1)}{2-1}=1>0,
$$

and the function $f$ is not decreasing.

Theorem 1. Let $I \subset \mathbb{R}$ be an interval. The functions $f, F: I \rightarrow \mathbb{R}$ satisfy the inequality (1.2) :

$$
\frac{f(x)-F(y)}{x-y} \geq 0, \quad x, y \in I, x \neq y,
$$

if and only if $f$ and $F$ are nondecreasing and $f(x)=F(x)$ at every point of the continuity of one of these function.

Proof. In view of Lemma 1 , the functions $f$ and $F$ are nondecreasing. Denote by $C_{f}$ the set of all continuity points of $f$. If $x \in C_{f}$ then, for all $s, t \in I$ such that $s<x<t$ in view of the first of inequalities of (1.2),

$$
f(s) \leq F(x) \leq f(t) .
$$

Letting here $s$ and $t$ tend to $x$, and using the continuity of $f$, we hence get $f(x)=F(x)$. If $x \in C_{F}$ we argue similarly. The converse implication is obvious.

Remark 1. Theorem 1 generalizes the classical criterion of the monotonicity of a differentiable function: the nonnegativity of derivative.

\section{Mean-value property for the arithmetic mean}

Theorem 2. Let $I \subset \mathbb{R}$ be a set such that card $I>3$. The functions $f, F, g, G$ : $I \rightarrow \mathbb{R}$, and satisfy the functional equation

$$
\frac{f(x)-F(y)}{x-y}=\mathcal{A}(g(x), G(y)), \quad x, y \in I, x \neq y
$$

if and only if

$$
f(x)=F(x)=\frac{a}{2} x^{2}+b x+d, \quad g(x)=G(x)=a x+b, \quad x \in I
$$

for some $a, b, c, d \in \mathbb{R}$. 
Proof. Assume that the functions $f, F, g, G$ satisfy the equation (2.1). Then by the definition of $\mathcal{A}$,

$$
f(x)-F(y)=\frac{g(x)+G(y)}{2}(x-y), \quad x, y \in I, x \neq y .
$$

Replacing here $y$ by $z$ we get

$$
f(x)-F(z)=\frac{g(x)+G(z)}{2}(x-z), \quad x, z \in I, x \neq z .
$$

Subtracting the respective sides of these two equations we obtain

$$
2[F(z)-F(y)]=[G(y)-G(z)] x+(z-y) g(x)+[z G(z)-y G(y)],
$$

whence

$$
g(x)=\frac{G(z)-G(y)}{z-y} x+\frac{2[F(z)-F(y)]-[z G(z)-y G(y)]}{z-y}
$$

for all $x, y, z \in I, y \neq x \neq z$. Since in this formula $y, z \in I, y \neq z$, can be here chosen arbitrarily, it follows that

$$
g(x)=a x+b, \quad x \in I
$$

for some $a, b \in \mathbb{R}$ such that $a^{2}+b^{2}>0$. Moreover the equation (2.3) implies that

$$
\frac{G(z)-G(y)}{z-y}=a, \quad y, z \in I, y \neq z,
$$

and

$$
2 \frac{F(z)-F(y)}{z-y}-\frac{z G(z)-y G(y)}{z-y}=b, \quad y, z \in I, y \neq z .
$$

Equality (2.5) implies that, for some real $c$,

$$
G(x)=a x+c, \quad x \in I .
$$

Since

$$
\frac{z G(z)-y G(y)}{z-y}=\frac{G(z)-G(y)}{z-y} z+G(y),
$$

from (2.6) and (2.7) we get

$$
2 \frac{F(z)-F(y)}{z-y}=a z+b, \quad y, z \in I, y \neq z,
$$

whence, obviously,

$$
F(x)=\frac{a}{2} x^{2}+b x+d, \quad x \in I
$$

for some real $d$. Setting the functions $g, G$ and $F$ given by (2.4), (2.7) and (2.8) into the equation (2.2) we get

$$
f(x)=\frac{a}{2} x^{2}+\frac{b+c}{2} x+d+\frac{b-c}{2} y, \quad x, y \in I, x \neq y .
$$


Since the function on the right-hand side cannot depend on $y$, it follows that

$$
c=b
$$

and, consequently,

$$
f(x)=\frac{a}{2} x^{2}+b x+d, \quad x \in I .
$$

Moreover, from (2.7) and (2.9) we get

$$
G(x)=a x+b, \quad x \in I .
$$

Since the functions $f$ given by $(2.10), F$ given by (2.8), $g$ given by (2.4) and $G$ given by (2.11) satisfy the equation $(2.1)$, the proof is completed.

\section{Mean-value property for the geometric mean}

Theorem 3. Let $I \subset \mathbb{R}$ be an interval. The functions $f, F: I \rightarrow \mathbb{R}$, and $g, G: I \rightarrow(0, \infty)$ satisfy the functional equation

$$
\frac{f(x)-F(y)}{x-y}=\mathcal{G}(g(x), G(y)), \quad x, y \in I, x \neq y,
$$

if and only if, one of the following cases occurs:

(i) there are $p, q, r \in \mathbb{R}, p>0, q>0$, such that

$$
f(x)=F(x)=p q x+r, \quad g(x)=p^{2}, \quad G(x)=q^{2}, \quad x \in I ;
$$

(ii) there are $p, q, r, s \in \mathbb{R}, p>0, q>0$, such that

$$
f(x)=F(x)=s-\frac{p q}{x+r}, \quad g(x)=\frac{p^{2}}{(x+r)^{2}}, \quad G(x)=\frac{q^{2}}{(x+r)^{2}}, \quad x \in I .
$$

Proof. If the functions $f, F, g, G$ satisfy the equation (3.1), then by the definition of $\mathcal{G}$,

$$
f(x)-F(y)=(x-y) \sqrt{g(x) G(y)}, \quad x, y \in I, x \neq y .
$$

Replacing here $y$ by $z$ we get

$$
f(x)-F(z)=(x-z) \sqrt{g(x) G(z)}, \quad x, z \in I, x \neq z .
$$

As the right-hand side of the equation (3.1) is positive, by Lemma 1 the function $F$ (as well as $f$ ) is one-to-one. Subtracting the respective sides of these two equations we obtain

$$
F(z)-F(y)=[(\sqrt{G(y)}-\sqrt{G(z)}) x+(z \sqrt{G(z)}-y \sqrt{G(y)})] \sqrt{g(x)},
$$

whence

$$
\sqrt{g(x)}=\frac{1}{\frac{\sqrt{G(y)}-\sqrt{G(z)}}{F(z)-F(y)} x+\frac{z \sqrt{G(z)}-y \sqrt{G(y)}}{F(z)-F(y)}}, \quad x, y, z \in I, y \neq x \neq z
$$


for all $x, y, z \in I, y \neq x \neq z$. Since $y, z \in I, y \neq z$, can be here arbitrarily fixed, it follows that

$$
g(x)=\frac{1}{(a x+b)^{2}}, \quad x \in I
$$

for some $a, b \in \mathbb{R}$ such that $a^{2}+b^{2}>0$. Now the equation (3.3) implies that

$$
\frac{\sqrt{G(y)}-\sqrt{G(z)}}{F(z)-F(y)}=a, \quad y, z \in I, y \neq z,
$$

and

$$
\frac{z \sqrt{G(z)}-y \sqrt{G(y)}}{F(z)-F(y)}=b, \quad y, z \in I, y \neq z .
$$

From (3.5) we have

$$
\sqrt{G(y)}+a F(y)=\sqrt{G(z)}+a F(z), \quad y, z \in I, y \neq z,
$$

which implies that, for some $k \in \mathbb{R}$,

$$
\sqrt{G(x)}+a F(x)=k, \quad x \in I .
$$

Similarly, from (3.6), we get

$$
x \sqrt{G(x)}-b F(x)=m, \quad x \in I .
$$

The last two equations imply that, for some $c>0$,

$$
G(x)=\frac{c^{2}}{(a x+b)^{2}}, \quad x \in I,
$$

and

$$
F(x)=\frac{n x+d}{a x+b}, \quad x \in I
$$

for some $n, d \in \mathbb{R}$. From (3.2) and (3.4) we obtain that

$$
f(x)=\frac{k x+m}{a x+b}, \quad x \in I
$$

for some $k, m \in \mathbb{R}$. Setting the functions (3.4), (3.7), (3.8) and (3.9) into the equation (3.2) we get

$$
a(k-n) x y-(a d-b k+c) x+(a m-b n+c) y+b(m-d)=0
$$

for all $x, y \in I, x \neq y$. It follows that

(3.10) $a(k-n)=0, \quad a d-b k+c=0, \quad a m-b n+c=0, \quad b(m-d)=0$.

If $a=0$, then $b \neq 0$, and this system of equations simplifies to

$$
b k-c=0, \quad b n-c=0, \quad m-d=0 .
$$

Hence

$$
k=n=\frac{c}{b}, \quad m=d,
$$


whence

$$
f(x)=F(x)=\frac{c}{b^{2}} x+\frac{d}{b}, \quad x \in I .
$$

In this case, by (3.4) and (3.7), the functions $g$ and $G$ are constant; more precisely, we have

$$
g(x)=\frac{1}{b^{2}}, \quad G(x)=\left(\frac{c}{b}\right)^{2}, \quad x \in I .
$$

Since $\frac{c}{b^{2}}=\frac{1}{b} \cdot \frac{c}{b}$, setting

$$
p:=\frac{1}{b^{2}}, \quad q:=\frac{c}{b}, \quad r:=\frac{d}{b},
$$

we obtain

$$
f(x)=F(x)=p q x+r, \quad g(x)=p^{2}, \quad G(x)=q^{2}, \quad x \in I .
$$

If $b=0$, then $a \neq 0$, and the system (3.10) becomes

$$
k-r=0, \quad a d+c=0, \quad a m+c=0 .
$$

Hence

whence

$$
r=k, \quad m=d=-\frac{c}{a},
$$

$$
f(x)=F(x)=\frac{k}{a}-\frac{c}{a^{2}} \frac{1}{x}, \quad x \in I .
$$

Replacing in his formula $k$ by ad we get

$$
f(x)=F(x)=d-\frac{c}{a^{2}} \frac{1}{x}, \quad x \in I .
$$

In this case, by (3.4) and (3.7), we have

$$
g(x)=\frac{1}{a^{2} x^{2}}, \quad G(x)=\frac{c^{2}}{a^{2} x^{2}}, \quad x \in I .
$$

Setting

we hence get

$$
p:=\frac{1}{a}, \quad q:=\frac{c}{a}, \quad s:=\frac{k}{a},
$$

$$
f(x)=F(x)=s-\frac{p q}{x}, \quad g(x)=\frac{p^{2}}{x^{2}}, \quad G(x)=\frac{q^{2}}{x^{2}}, \quad x \in I .
$$

If $a b \neq 0$, the from $(3.10)$ we obtain

$$
n=k=\frac{a d+c}{b}, \quad m=d,
$$

whence

$$
f(x)=F(x)=\frac{c}{b} \frac{x}{a x+b}+\frac{d}{b}=d+\frac{c}{a b}-\frac{c}{a^{2}} \frac{1}{x+\frac{b}{a}}, \quad x \in I .
$$


From (3.4) and (3.7) we have

$$
g(x)=\frac{1}{a^{2}} \frac{1}{\left(x+\frac{b}{a}\right)^{2}}, \quad G(x)=\frac{c^{2}}{a^{2}} \frac{1}{\left(x+\frac{b}{a}\right)^{2}}, \quad x \in I .
$$

Therefore, setting

$$
p:=\frac{1}{a}, \quad q:=\frac{c}{a}, \quad r:=\frac{b}{a}, \quad s:=d+\frac{c}{a b},
$$

we hence obtain

$f(x)=F(x)=s-\frac{p q}{x+r}, \quad g(x)=\frac{p^{2}}{(x+r)^{2}}, \quad G(x)=\frac{q^{2}}{(x+r)^{2}}, \quad x \in I$.

Note that taking here $r=0$ we get the formulas obtained in the previous case. Since the geometric mean is a positive function, (3.1) and Lemma 1 imply that $f$ is strictly increasing. It follows that $p$ and $q$ has to be of the same sign. Of course, we can assume that both are positive. Since in each of these three cases, the functions $f, F, g, G$ satisfy the equation (3.1), the proof is completed.

\section{Mean-value property for the harmonic mean}

Theorem 4. Let $I \subset \mathbb{R}$ be an arbitrary set such that card $I>3$. The functions $f, F: I \rightarrow \mathbb{R}$, and $g, G: I \rightarrow(0, \infty)$ satisfy the functional equation

$$
\frac{f(x)-F(y)}{x-y}=\mathcal{H}(g(x), G(y)), \quad x, y \in I, x \neq y,
$$

if and only if, one of the following cases occurs:

(i) there are $p, q \in \mathbb{R}, p>0$, such that

$$
f(x)=F(x)=p x+q, \quad g(x)=G(x)=p, \quad x \in I ;
$$

(ii) there are $p, q, r \in \mathbb{R}, p>0$, such that

$$
f(x)=F(x)=2 p \sqrt{x+q}+r, \quad g(x)=G(x)=\frac{p}{\sqrt{x+q}}, \quad x \in I ;
$$

(iii) there are $p, q, r \in \mathbb{R}, p>0$, such that

$$
f(x)=F(x)=2 p \sqrt{q-x}+r, \quad g(x)=G(x)=\frac{p}{\sqrt{q-x}} \quad x \in I .
$$

Proof. Assume that the functions $f, F, g, G: I \rightarrow \mathbb{R}$ satisfy the equation (4.1). Since the right-hand side of the equation (4.1) is positive, Theorem 1 implies that the functions $f$ and $F$ are strictly increasing and

$$
F(x)=f(x), \quad x \in J:=C_{f} \cup C_{F},
$$

where $C_{f}$ denotes the set of all continuity points of $f$. Thus, from (4.1) we get

$$
\frac{f(x)-f(y)}{x-y}=\mathcal{H}(g(x), G(y)), \quad x, y \in I, x \neq y
$$


whence, by the definition of $\mathcal{H}$, we get

$$
\frac{x-y}{f(x)-f(y)}=\frac{\frac{1}{g(x)}+\frac{1}{G(y)}}{2}, \quad x, y \in I, x \neq y .
$$

Take arbitrary $u, v \in f(J)$. Setting $x:=f^{-1}(u), y:=f^{-1}(v)$ in this equation we get

$$
\frac{f^{-1}(u)-f^{-1}(v)}{u-v}=\frac{\frac{1}{g \circ f^{-1}(u)}+\frac{1}{G \circ f^{-1}(v)}}{2}, \quad u, v \in f(J), u \neq v .
$$

Since, obviously, card $f(J)=$ card $J>3$, applying Theorem 2 with $I$ replaced by $f(J) ; f$ and $F$ replaced by $f^{-1}, g$ replaced by $\frac{1}{g \circ f^{-1}}$ and $G$ replaced by $\frac{1}{G \circ f^{-1}}$ we conclude that there are $a, b, c, d \in \mathbb{R}$ such that, for some $a, b, c, d \in \mathbb{R}$.,

$$
f^{-1}(u)=\frac{a}{2} u^{2}+b u+d, \quad u \in f(J),
$$

and

$$
\frac{1}{g \circ f^{-1}(u)}=\frac{1}{G \circ f^{-1}(u)}=a u+b, \quad u \in f(J) .
$$

If $a \neq 0$ we hence we get that, for every $x \in J$, either

$$
f(x)=-\frac{b}{a}-\frac{1}{a} \sqrt{2 a x+\left(b^{2}-2 a d\right)} \text { and } g(x)=G(x)=-\frac{1}{\sqrt{2 a x+\left(b^{2}-2 a d\right)}}
$$

or

$$
f(x)=-\frac{b}{a}+\frac{1}{a} \sqrt{2 a x+\left(b^{2}-2 a d\right)} \text { and } g(x)=G(x)=\frac{1}{\sqrt{2 a x+\left(b^{2}-2 a d\right)}} .
$$

Note that the first of these formulas must be omitted, as $g$ (and $G$ ) has positive values. Since, by Lemma $1, f$ is increasing in the interval $I$, the set $J$ is dense in the interval $I$, and

$$
f(x)=-\frac{b}{a}+\frac{1}{a} \sqrt{2 a x+\left(b^{2}-2 a d\right)}, \quad x \in J,
$$

it follows that $J=I$. Hence, by (4.2),

$$
f(x)=F(x)=-\frac{b}{a}+\frac{1}{a} \sqrt{2 a x+\left(b^{2}-2 a d\right)}, \quad x \in I,
$$

and, by (4.4)

$$
g(x)=G(x)=\frac{1}{\sqrt{2 a x+\left(b^{2}-2 a d\right)}}, \quad x \in I .
$$

Assume that $a>0$. Setting here

we obtain

$$
p:=\frac{1}{\sqrt{2 a}}, \quad q:=\frac{b^{2}-2 a d}{2 a}, \quad r:=-\frac{b}{a},
$$

$$
f(x)=F(x)=2 p \sqrt{x+q}+r, \quad g(x)=G(x)=\frac{p}{\sqrt{x+q}} \quad x \in I,
$$


where $p>0$ and $q, r \in \mathbb{R}$ are arbitrary.

If $a<0$, setting

$$
p:=\frac{1}{\sqrt{-2 a}}, \quad q:=\frac{2 a d-b^{2}}{2 a}, \quad r:=-\frac{b}{a},
$$

we obtain

$$
f(x)=F(x)=2 p \sqrt{q-x}+r, \quad g(x)=G(x)=\frac{p}{\sqrt{q-x}} \quad x \in I,
$$

where $p>0$ and $q, r \in \mathbb{R}$ are arbitrary.

Now assume that $a=0$. In view of (4.3),

$$
f^{-1}(u)=b u+d, \quad u \in f(J) .
$$

Since $f$ is strictly increasing, it follows that $b>0$ and

$$
f(x)=F(x)=\frac{1}{b} x-\frac{d}{b}, \quad x \in I,
$$

and, by (4.4),

$$
g(x)=G(x)=\frac{1}{b}, \quad x \in I .
$$

Setting $p:=\frac{1}{b}$ and $q:=-\frac{d}{b}$ we obtain

$$
f(x)=F(x)=p x+q, \quad g(x)=G(x)=p, \quad x \in I,
$$

where $p>0$ and $q \in \mathbb{R}$ are arbitrary.

\section{Remark concerning on a relevant functional equation with five unknown functions}

From Theorem 2 we obtain the following:

Corollary 1. Let $X$ be a set such that card $X>3$ and let $f, F, g, G, h: X \rightarrow \mathbb{R}$. Suppose that $h$ is one-to-one. The functions $f, F, g, G, h$ satisfy the functional equation

$$
\frac{f(x)-F(y)}{h(x)-h(y)}=\mathcal{A}(g(x), G(y)), \quad x, y \in X, x \neq y
$$

if and only if

$$
f(x)=F(x)=\frac{a}{2} h(x)^{2}+b h(x)+d, \quad g(x)=G(x)=a h(x)+b, \quad x \in I
$$

for some $a, b, c, d \in \mathbb{R}$.

To prove it is enough to apply Theorem 1 to the functional equation $\frac{f \circ h^{-1}(u)-F \circ h^{-1}(u)}{u-v}=\mathcal{A}\left(g \circ h^{-1}(u), G \circ h^{-1}(v)\right), \quad u, v \in h(X), u \neq v$.

In a similar way one could formulate respective generalizations of Theorems 3 and 4 . 


\section{References}

[1] J. Aczél, A mean-value property of the derivative of quadratic polynomials - without mean values and derivatives, Math. Mag. 58 (1985), no. 1, 42-45.

[2] J. Aczél and M. Kuczma, On two mean value properties and functional equations associated with them, Aequationes Math. 38 (1989), no. 2-3, 216-235.

[3] M. Kuczma, On the quasiarithmetic mean in a mean value property and the associated functional equation, Aequationes Math. 41 (1991), no. 1, 33-54.

[4] J. Matkowski, A mean-value theorem and its applications, J. Math. Anal. Appl. 373 (2011), no. 1, 227-234.

5] , Power means generated by some mean-value theorems, Proc. Amer. Math. Soc. 139 (2011), no. 10, 3601-3610.

Faculty of Mathematics Computer Science and Econometrics

University of Zielona Góra

Szafrana 4A, PL-65-516 Zielona Góra, Poland

E-mail address: J.Matkowski@wmie.uz.zgora.pl 